\title{
New meson spectroscopy with open charm and beauty
}

\author{
P. Colangelo ${ }^{a}$, F. De Fazio ${ }^{a}$, F. Giannuzzi ${ }^{a, b}$ and S. Nicotri ${ }^{a, b}$ \\ ${ }^{a}$ Istituto Nazionale di Fisica Nucleare, Sezione di Bari, Italy \\ ${ }^{b}$ Dipartimento di Fisica, Università degli Studi di Bari, Italy
}

\begin{abstract}
All the available experimental information on open charm and beauty mesons is used to classify the observed states in heavy quark doublets. The masses of some of the still unobserved states are predicted, in particular in the beauty sector. Adopting an effective Lagrangian approach based on the heavy quark and chiral symmetry, individual decay rates and ratios of branching fractions are computed, with results useful to assign the quantum numbers to recently observed charmed states which still need to be properly classified. Implications and predictions for the corresponding beauty mesons are provided. The experimental results are already copious, and are expected to grow up thanks to the experiments at the LHC and to the future high-luminosity flavour and $p-\bar{p}$ facilities.
\end{abstract}

PACS numbers: 13.25.Ft,13.25.Hw, 12.39.Fe, 12.39.Hg

\section{INTRODUCTION}

The analysis of hadrons containing a single heavy quark can be afforded in a predictive framework exploiting the heavy quark (HQ) limit, which is formalized in the heavy quark effective theory (HQET) [1]. This is an effective theory formulated for $N_{f}$ heavy quarks $Q$ with mass $m_{Q} \gg \Lambda_{Q C D}$ (a condition generally denoted as the $m_{Q} \rightarrow \infty$ limit), keeping the four-velocity of $Q$ fixed. The theory displays heavy quark spin-flavour symmetries, i.e. invariance under $S U\left(2 N_{f}\right)$ transformations. Within this framework it is possible to describe several heavy hadron properties, the prime example being the relations among semileptonic transition form factors in weak heavy hadron matrix elements 2]. In this paper we consider another sector in which the heavy quark limit provides powerful predictions, the heavy meson spectroscopy [3]. The renewed interest in this subject is due to the remarkable number of resonances discovered in the recent years, a set of states that will presumably become richer with the data taking of the experiments at the CERN LHC, as well as of the new high-luminosity flavour factories and $p-\bar{p}$ facilities.

The classification of heavy $Q \bar{q}$ mesons ( $q$ being a light quark) in the HQ limit relies on the decoupling of the heavy quark spin from the spin of light degrees of freedom, the light antiquark and the gluons. The spin $s_{Q}$ of the heavy quark and the total angular momentum of the light degrees of freedom $s_{\ell}$ are separately conserved in strong interaction processes. Heavy mesons can therefore be classified according to the value of $s_{\ell}$, and can be collected in doublets; the two states of each doublet have total spin $J=s_{\ell} \pm \frac{1}{2}$ and parity $P=(-1)^{\ell+1}$, with $\ell$ the orbital angular momentum of the light degrees of freedom and $\vec{s}_{\ell}=\vec{\ell}+\vec{s}_{q}$ ( $s_{q}$ is the light antiquark spin). We refer to the two states in the same doublet as the spin partners. Since the properties of hadron states are independent of the spin and flavour of the heavy quark, the two states within each doublet are degenerate in the HQ limit and, due to flavour symmetry, the properties of the states in a doublet can be related to those of the corresponding states that differ for the flavour of the heavy quark. In practice, HQET turns out to be a theory of charmed and beauty hadrons, the top quark is too heavy to hadronize before decaying.

In the following we focus on the meson doublets corresponding to $\ell=0,1,2$ (in the quark model they are referred to as $s-, p-$ and $d$-wave states), we discuss their properties in the HQ limit and include corrections to the degeneracy condition within each doublet. This allows us to study how the observed charmed and beauty mesons fit in the theoretical classification. Furthermore, using the available data in the charm sector, the properties of the corresponding beauty mesons, if not yet observed, can be predicted.

Since several recently discovered states require a proper classification, it is useful to analyze strong decays of heavy mesons to light pseudoscalar mesons, because the decay rates of these processes depend on the quantum numbers of the decaying resonances. For such a purpose, we exploit an effective Lagrangian approach in which the heavy quark doublets are represented by effective fields, while the octet of light pseudo Goldstone mesons is grouped in a single field. The Lagrangian terms describing the strong decays of a heavy meson with the emission of a light pseudoscalar meson are invariant both under heavy quark spin-flavour transformations and chiral transformations of the light pseudo Goldstone boson fields.

In the exact HQ limit for the heavy meson doublets, considering the strong decays with a light pseudoscalar meson in the final state, as a consequence of the spin flavour symmetries, the following properties are expected [3]:

- The two states within a doublet are degenerate in mass;

- The two states within a doublet have the same full width; 
- The sum of the partial widths of a state in a doublet to another heavy state in another doublet with emission of a light meson is the same for the two states of a doublet;

- Spin symmetry predicts the ratios of partial decay widths for a given state;

- Partial decay widths are independent of the heavy quark flavour;

- Mass splittings among the different doublets are also independent of the heavy quark flavour.

In the following Sections we analyze the complete set of the established and newly observed open charm and beauty mesons, testing the above properties and discussing the possible deviations, with the aim of a unique description for all the experimental findings. On the basis of such a description, several predictions for still unobserved resonances, in particular in the beauty sector, will be obtained.

\section{HEAVY MESON DOUBLETS: AN OVERVIEW}

We discuss the states of the doublets corresponding to $\ell=0,1,2$. The lowest lying $Q \bar{q}$ mesons correspond to $\ell=0$, then $s_{\ell}^{P}=\frac{1}{2}^{-}$; this doublet consists of two states with spin-parity $J^{P}=\left(0^{-}, 1^{-}\right)$that we denote as $\left(P, P^{*}\right)$. For $\ell=1$ it could be either $s_{\ell}^{P}=\frac{1}{2}^{+}$or $s_{\ell}^{P}=\frac{3}{2}^{+}$. The two corresponding doublets have $J^{P}=\left(0^{+}, 1^{+}\right)$and $J^{P}=\left(1^{+}, 2^{+}\right)$. We denote the members of the $J_{s_{\ell}}^{P}=\left(0^{+}, 1^{+}\right)_{1 / 2}$ doublet as $\left(P_{0}^{*}, P_{1}^{\prime}\right)$, and those of the $J_{s_{\ell}}^{P}=\left(1^{+}, 2^{+}\right)_{3 / 2}$ doublet as $\left(P_{1}, P_{2}^{*}\right) . \quad \ell=2$ corresponds to either $s_{\ell}^{P}=\frac{3}{2}^{-}$or $s_{\ell}^{P}=\frac{5}{2}^{-}$; the states belonging to such doublets are denoted as $\left(P_{1}^{*}, P_{2}\right)$ and $\left(P_{2}^{\prime *}, P_{3}\right)$, respectively. We use an analogous notation for the radial excitations of these states with radial quantum number $n=2$, distinguishing their fields by a tilde $\left(\tilde{P}, \tilde{P}^{*}, \ldots\right)$.

The expressions for the effective fields describing the various doublets in the HQ limit are collected below: $H_{a}$ ( $a=u, d, s$ a light flavour index) corresponds to $s_{\ell}^{P}=\frac{1}{2}^{-} ; S_{a}$ and $T_{a}$ to $s_{\ell}^{P}=\frac{1}{2}^{+}$and $s_{\ell}^{P}=\frac{3}{2}^{+}$, respectively; $X_{a}$ describes the doublet with $s_{\ell}^{P}=\frac{3}{2}^{-}$, and $X_{a}^{\prime}$ the $s_{\ell}^{P}=\frac{5}{2}^{-}$doublet:

$$
\begin{aligned}
H_{a} & =\frac{1+\not}{2}\left[P_{a \mu}^{*} \gamma^{\mu}-P_{a} \gamma_{5}\right] \\
S_{a} & =\frac{1+\chi}{2}\left[P_{1 a}^{\prime \mu} \gamma_{\mu} \gamma_{5}-P_{0 a}^{*}\right] \\
T_{a}^{\mu} & =\frac{1+\not}{2}\left\{P_{2 a}^{\mu \nu} \gamma_{\nu}-P_{1 a \nu} \sqrt{\frac{3}{2}} \gamma_{5}\left[g^{\mu \nu}-\frac{1}{3} \gamma^{\nu}\left(\gamma^{\mu}-v^{\mu}\right)\right]\right\} \\
X_{a}^{\mu} & =\frac{1+\not}{2}\left\{P_{2 a}^{* \mu \nu} \gamma_{5} \gamma_{\nu}-P_{1 a \nu}^{\prime *} \sqrt{\frac{3}{2}}\left[g^{\mu \nu}-\frac{1}{3} \gamma^{\nu}\left(\gamma^{\mu}+v^{\mu}\right)\right]\right\} \\
X_{a}^{\prime \mu \nu} & =\frac{1+\not}{2}\left\{P_{3 a}^{\mu \nu \sigma} \gamma_{\sigma}-P_{2 a}^{*^{\prime} \alpha \beta} \sqrt{\frac{5}{3}} \gamma_{5}\left[g_{\alpha}^{\mu} g_{\beta}^{\nu}-\frac{1}{5} \gamma_{\alpha} g_{\beta}^{\nu}\left(\gamma^{\mu}-v^{\mu}\right)-\frac{1}{5} \gamma_{\beta} g_{\alpha}^{\mu}\left(\gamma^{\nu}-v^{\nu}\right)\right]\right\} .
\end{aligned}
$$

The various operators in Eq.(11) annihilate mesons of four velocity $v$, which is conserved in strong interaction processes; they include a factor $\sqrt{m_{Q}}$ and have dimension 3/2. The octet of light pseudoscalar mesons is introduced through the definitions $\xi=e^{\frac{i \mathcal{M}}{f_{\pi}}}$ and $\Sigma=\xi^{2}$, with the matrix $\mathcal{M}$ incorporating the $\pi, K$ and $\eta$ fields $\left(f_{\pi}=132 \mathrm{MeV}\right)$ :

$$
\mathcal{M}=\left(\begin{array}{ccc}
\sqrt{\frac{1}{2}} \pi^{0}+\sqrt{\frac{1}{6}} \eta & \pi^{+} & K^{+} \\
\pi^{-} & -\sqrt{\frac{1}{2}} \pi^{0}+\sqrt{\frac{1}{6} \eta} & K^{0} \\
K^{-} & \bar{K}^{0} & -\sqrt{\frac{2}{3}} \eta
\end{array}\right) .
$$

With these fields an effective Lagrangian can be written, invariant under heavy quark spin-flavour and light quark chiral transformations [4, 5]. The kinetic terms of the heavy meson doublets and of the $\Sigma$ field read:

$$
\begin{aligned}
\mathcal{L} & =i \operatorname{Tr}\left[\bar{H}_{b} v^{\mu} D_{\mu b a} H_{a}\right]+\frac{f_{\pi}^{2}}{8} \operatorname{Tr}\left[\partial^{\mu} \Sigma \partial_{\mu} \Sigma^{\dagger}\right] \\
& +\operatorname{Tr}\left[\bar{S}_{b}\left(i v^{\mu} D_{\mu b a}-\delta_{b a} \Delta_{S}\right) S_{a}\right]+\operatorname{Tr}\left[\bar{T}_{b}^{\alpha}\left(i v^{\mu} D_{\mu b a}-\delta_{b a} \Delta_{T}\right) T_{a \alpha}\right] \\
& +\operatorname{Tr}\left[\bar{X}_{b}^{\alpha}\left(i v^{\mu} D_{\mu b a}-\delta_{b a} \Delta_{X}\right) X_{a \alpha}\right]+\operatorname{Tr}\left[\bar{X}_{b}^{\prime \alpha \beta}\left(i v^{\mu} D_{\mu b a}-\delta_{b a} \Delta_{X^{\prime}}\right) X_{a \alpha \beta}^{\prime}\right] .
\end{aligned}
$$


Such terms involve the operators $D$ and $\mathcal{A}$ :

$$
\begin{aligned}
D_{\mu b a} & =-\delta_{b a} \partial_{\mu}+\mathcal{V}_{\mu b a}=-\delta_{b a} \partial_{\mu}+\frac{1}{2}\left(\xi^{\dagger} \partial_{\mu} \xi+\xi \partial_{\mu} \xi^{\dagger}\right)_{b a} \\
\mathcal{A}_{\mu b a} & =\frac{i}{2}\left(\xi^{\dagger} \partial_{\mu} \xi-\xi \partial_{\mu} \xi^{\dagger}\right)_{b a} .
\end{aligned}
$$

The mass parameters $\Delta_{F}$ (with $F=S, T, X, X^{\prime}$ ) represent the mass splittings between the higher mass doublets and the lowest lying doublet described by the field $H$; they can be expressed in terms of the spin-averaged masses of the doublets:

$$
\Delta_{F}=\bar{M}_{F}-\bar{M}_{H}
$$

with

$$
\begin{aligned}
\bar{M}_{H} & =\frac{3 M_{P^{*}}+M_{P}}{4} \\
\bar{M}_{S} & =\frac{3 M_{P_{1}^{\prime}}+M_{P_{0}^{*}}}{4} \\
\bar{M}_{T} & =\frac{5 M_{P_{2}^{*}}+3 M_{P_{1}}}{8} \\
\bar{M}_{X} & =\frac{5 M_{P_{2}}+3 M_{P_{1}^{*}}}{8} \\
\bar{M}_{X^{\prime}} & =\frac{7 M_{P_{3}}+5 M_{P_{2}^{\prime *}}}{12} .
\end{aligned}
$$

Corrections to the heavy quark limit are represented by symmetry breaking terms suppressed by increasing powers of the inverse heavy quark mass $m_{Q}$ []. $]$. In particular, the mass degeneracy between the members of the meson doublets is broken by the terms:

$$
\begin{aligned}
\mathcal{L}_{1 / m_{Q}}= & \frac{1}{2 m_{Q}}\left\{\lambda_{H} \operatorname{Tr}\left[\bar{H}_{a} \sigma^{\mu \nu} H_{a} \sigma_{\mu \nu}\right]+\lambda_{S} \operatorname{Tr}\left[\bar{S}_{a} \sigma^{\mu \nu} S_{a} \sigma_{\mu \nu}\right]+\lambda_{T} \operatorname{Tr}\left[\bar{T}_{a}^{\alpha} \sigma^{\mu \nu} T_{a}^{\alpha} \sigma_{\mu \nu}\right]\right. \\
& \left.+\lambda_{X} \operatorname{Tr}\left[\bar{X}_{a}^{\alpha} \sigma^{\mu \nu} X_{a \alpha} \sigma_{\mu \nu}\right]+\lambda_{X^{\prime}} \operatorname{Tr}\left[\bar{X}_{a}^{\prime \alpha \beta} \sigma^{\mu \nu} X_{a}^{\prime \alpha \beta} \sigma_{\mu \nu}\right]\right\}
\end{aligned}
$$

with the constants $\lambda_{H}, \lambda_{S}, \lambda_{T}, \lambda_{X}$ and $\lambda_{X^{\prime}}$ connected to the hyperfine splitting in each doublet:

$$
\begin{aligned}
\lambda_{H} & =\frac{1}{8}\left(M_{P^{*}}^{2}-M_{P}^{2}\right) \\
\lambda_{S} & =\frac{1}{8}\left(M_{P_{1}^{\prime}}^{2}-M_{P_{0}^{*}}^{2}\right) \\
\lambda_{T} & =\frac{3}{16}\left(M_{P_{2}^{*}}^{2}-M_{P_{1}}^{2}\right) \\
\lambda_{X} & =\frac{3}{16}\left(M_{P_{2}}^{2}-M_{P_{1}^{*}}^{2}\right) \\
\lambda_{X^{\prime}} & =\frac{5}{24}\left(M_{P_{3}}^{2}-M_{P_{2}^{\prime *}}^{2}\right) .
\end{aligned}
$$

In Table \ we collect the observed charmed $c \bar{q}$ and $c \bar{s}$, and beauty $b \bar{q}$ and $b \bar{s}$ (with $q=u, d$ ) mesons, with a classification established within the heavy quark doublet scheme. In the Table we also include states not yet classified: Their position reflects our proposed assignment of their quantum numbers, and to indicate this (still unsettled) situation we put a $\star$ mark in correspondence of such mesons. Alternative classifications will also be discussed in the following.

The states belonging to the lowest $s_{\ell}^{P}=\frac{1}{2}^{-}$and $n=1$ doublet are well recognized, hence our discussion mainly concerns the excited doublets with either $\ell=\frac{1}{2}^{+}, \frac{3}{2}^{ \pm}, \ldots$, or $n>1$. In Tables III and IIII we collect the values of the masses and widths of such resonances as reported by the Particle Data Group (PDG) 7]. For the masses of the states of the lightest doublet, as well as for the masses of the light pseudoscalar mesons, we refer to the PDG values.

Let us examine the various entries in Table II The $s_{\ell}^{P}=\frac{3}{2}^{+}$charmed doublets are filled by the states $\left(D_{1}(2420), D_{2}^{*}(2460)\right)$ and $\left(D_{s 1}(2536), D_{s 2}^{*}(2573)\right)$ in the non-strange and strange case, respectively. Considering 
their widths in Table II can be noticed that these states are quite narrow, in accordance with the expectation, since their strong decays occur in $d$-wave: the widths of strong decays to a light pseudoscalar meson of momentum $\vec{p}$ are proportional to $|\vec{p}|^{2 \ell+1}$, with $\ell$ the angular momentum transferred in the decay. In these modes $|\vec{p}|$ is small, and highest values of $\ell$ correspond to the largest suppression of the decay rate.

The states $\left(D_{0}^{*}(2400), D_{1}^{\prime}(2430)\right)$ and $\left(D_{s 0}^{*}(2317), D_{s 1}^{\prime}(2460)\right)$ can be identified with the members of the $s_{\ell}^{P}=\frac{1}{2}^{+}$ charm doublet, although they present puzzling features. The non strange states follow the expectation of being broad, their strong decays occurring in $s$-wave. Evidences of $c \bar{q}$ broad states were provided by CLEO [8], Belle [9] and FOCUS [10] collaborations, but the separate identification of the two states, together with measurement of their masses and widths, is due to Belle [9]. The strange partners, first observed in 2003 [11], are very narrow in contrast to expectations. This feature can be attributed to their masses below the $D K$ (for $D_{s 0}^{*}(2317)$ ) and $D^{*} K$ (for $D_{s 1}^{\prime}(2460)$ ) thresholds, so that the isospin-conserving decays are kinematically forbidden. The observed strong decays to $D_{s} \pi^{0}$ and $D_{s}^{*} \pi^{0}$ violate the isospin conservation, hence the narrow widths. Their identification with the doublet $\left(D_{s 0}^{*}, D_{s 1}^{\prime}\right)$ is supported by a light-cone QCD sum rule analysis [12] which reproduces the experimentally observed hierarchy of the radiative decay modes 7]. A puzzling aspect is the mass degeneracy between the strange states and their non strange partners ${ }^{1}$. Another issue is the possible mixing between the two $1^{+}$states: in the case of non strange mesons, the Belle collaboration has determined the mixing angle $\theta$, with the result: $\theta=-0.10 \pm 0.03 \pm 0.02 \pm 0.02 \mathrm{rad}$ [9], indicating a small mixing.

The other entries in Table 1 correspond to the most recent observations. $D_{s J}(2860)$ was observed by the BaBar collaboration [14], and $D_{s 1}^{*}(2700)$ by the Belle [15] and BaBar [14] collaborations, both the resonances in the $D K$ final state. The two resonances have been confirmed in $p p$ collisions at the LHC [16]. The spin-parity $J^{P}=1^{-}$of $D_{s 1}^{*}(2700)$ has been established studying the production in $B$ decays. $D_{s 1}^{*}(2700)$ and $D_{s J}(2860)$ are also seen to decay to $D^{*} K$ [17], hence they have natural parity $J^{P}=1^{-}, 2^{+}, 3^{-}, \ldots$. The $D^{*} K$ mode excludes the assignment $J^{P}=0^{+}$ for $D_{s J}(2860)$. Additional information comes from the measurements of the ratios of decay rates

$$
\begin{aligned}
& \frac{B R\left(D_{s 1}^{*}(2700) \rightarrow D^{*} K\right)}{B R\left(D_{s 1}^{*}(2700) \rightarrow D K\right)}=0.91 \pm 0.13_{\text {stat }} \pm 0.12_{\text {syst }} \\
& \frac{B R\left(D_{s J}(2860) \rightarrow D^{*} K\right)}{B R\left(D_{s J}(2860) \rightarrow D K\right)}=1.10 \pm 0.15_{\text {stat }} \pm 0.19_{\text {syst }},
\end{aligned}
$$

where $D^{(*)} K$ is the sum over the final states $D^{(*) 0} K^{+}$and $D^{(*)^{+}} K_{S}^{0}$ [17]. Comparing these data with the predictions obtained in the heavy quark limit [18], we argue that $D_{s 1}^{*}(2700)$ is the first radial excitation of $D_{s}^{*}(2112)$.

The case of $D_{s J}(2860)$ is more uncertain. For the possible quantum number assignments to $D_{s J}(2860)$, one can follow the discussion in [19]. Since this resonance decays to both $D K$ and $D^{*} K$, it may be identified with the lowest lying $n=1$ state with either $J_{s_{\ell}}^{P}=1_{3 / 2}^{-}$, i.e. $D_{s 1}^{*}$ in the $X$ doublet, or $J_{s_{\ell}}^{P}=3_{5 / 2}^{-}$, i.e. the state $D_{s 3}$ in the $X^{\prime}$ doublet. Another possibility is the identification with the radial excitation with $n=2$ and $J_{s_{\ell}}^{P}=2_{1 / 2}^{+}$, i.e. the state $\tilde{D}_{s 2}^{*}$ in

Table I: Observed open charm and open beauty mesons, classified in HQ doublets. States with uncertain assignment are

\begin{tabular}{|c|c|c|c|c|c|c|c|c|c|c|}
\hline doublet & $s_{\ell}^{P}$ & $J^{P}$ & $c \bar{q} \quad(\mathrm{n}=1)$ & $c \bar{q}(\mathrm{n}=2)$ & $c \bar{s} \quad(\mathrm{n}=1)$ & $c \bar{s} \quad(\mathrm{n}=2)$ & $b \bar{q} \quad(\mathrm{n}=1)$ & $b \bar{q}(\mathrm{n}=2)$ & $b \bar{s} \quad(\mathrm{n}=1)$ & $b \bar{s} \quad(\mathrm{n}=2)$ \\
\hline \multirow{2}{*}{$H$} & \multirow[b]{2}{*}{$\frac{1}{2}$} & $0^{-}$ & $D(1869)$ & $D(2550) \star$ & $D_{s}(1968)$ & & $B(5279)$ & & $B_{s}(5366)$ & \\
\hline & & $1^{-}$ & $D^{*}(2010)$ & $D^{*}(2600) \star$ & $D_{s}^{*}(2112)$ & $D_{s 1}^{*}(2700)$ & $B^{*}(5325)$ & & $B_{s}^{*}(5415)$ & \\
\hline \multirow{2}{*}{$S$} & \multirow{2}{*}{$\frac{1}{2}^{+}$} & $0^{+}$ & $D_{0}^{*}(2400)$ & & $D_{s 0}^{*}(2317)$ & & & & & \\
\hline & & $1^{+}$ & $D_{1}^{\prime}(2430)$ & & $D_{s 1}^{\prime}(2460)$ & $D_{s J}(3040) \star$ & & & & \\
\hline \multirow{2}{*}{$T$} & \multirow{2}{*}{$\frac{3}{2}^{+}$} & $1^{+}$ & $D_{1}(2420)$ & & $D_{s 1}(2536)$ & $D_{s J}(3040) \star$ & $B_{1}(5721)$ & & $B_{s 1}(5830)$ & \\
\hline & & $2^{+}$ & $D_{2}^{*}(2460)$ & & $D_{s 2}^{*}(2573)$ & & $B_{2}^{*}(5747)$ & & $B_{s 2}^{*}(5840)$ & \\
\hline \multirow{2}{*}{$X$} & \multirow{2}{*}{$\frac{3}{2}^{-}$} & $1^{-}$ & & & & & & & & \\
\hline & & $2^{-}$ & & & & & & & & \\
\hline \multirow{2}{*}{$X^{\prime}$} & \multirow{2}{*}{$\frac{5}{2}^{-}$} & $2^{-}$ & $D(2750) \star$ & & & & & & & \\
\hline & & $3^{-}$ & $D(2760) \star$ & & $D_{s J}(2860)$ & & & & & \\
\hline
\end{tabular}
indicated with $\star$ and classified according to the scheme proposed in this study.

\footnotetext{
1 The mixing between a $c \bar{s}$ and a four quark configuration could be invoked as an explanation of this degeneracy [13].
} 
the $\tilde{T}$ doublet. Allowed decay modes are into $D K, D_{s} \eta, D^{*} K$ and $D_{s}^{*} \eta$. From the ratios of strong decay rates in the three possible cases, the identification with $D_{s 3}$ was proposed [19]. An important argument concerns the decay width, since $D_{s 3}$ is expected to decay in $f$ - wave, which would explain the observed narrow width of the resonance. $D_{s 1}^{*}$ and $\tilde{D}_{s 2}^{*}$ decay in $p$ - and $d$ - wave, respectively, which makes the identification of $D_{s J}(2860)$ with $D_{s 1}^{*}$ unlikely, but does not exclude that with $\tilde{D}_{s 2}^{*}$. Using typical values for the strong decay constant governing its strong decays, it turns out that indeed $D_{s 1}^{*}$ should have a width incompatible with the experimental findings. As for $\tilde{D}_{s 2}^{*}$, its mass is expected to be larger on the basis of potential model calculations: actually, the prediction $M\left(\tilde{D}_{s 2}^{*}\right) \simeq 3.157 \mathrm{GeV}$ [20] must be contrasted to $M\left(D_{s 3}\right) \simeq 2.925 \mathrm{GeV}$. As a conclusion, we continue to propose for $D_{s J}(2860)$ the assignment $J^{P}=3^{-}$with $n=1$. The alternative identification with $\tilde{D}_{s 2}^{*}$ is discussed in the subsequent Sections.

The BaBar collaboration observed another broad structure in the $D^{*} K$ invariant mass distribution, $D_{s J}(3040)$, with too limited statistics to permit studies of angular distributions for this state [17]. Since $D_{s J}(3040)$ decays to $D^{*} K$ and it is not found in the $D K$ distribution, it has unnatural parity $J^{P}=1^{+}, 2^{-}, 3^{+}, \cdots$. The lightest not yet observed states with these quantum numbers are the two $J^{P}=2^{-}$states belonging to the $\ell=2$ doublets, $D_{s 2}$ with $s_{\ell}^{P}=\frac{3}{2}^{-}$and $D_{s 2}^{\prime *}$ with $s_{\ell}^{P}=\frac{5}{2}^{-}$. The case $J^{P}=3^{+}$corresponds to a doublet with $s_{\ell}^{P}=\frac{7}{2}^{+}$, the mass of which is expected to be larger. In the case of radial excitations, the identification with the states with $n=2, J^{P}=1^{+}$, and $s_{\ell}^{P}=\frac{1}{2}^{+}$(the meson $\tilde{D}_{s 1}^{\prime}$ ) or $s_{\ell}^{P}=\frac{3}{2}^{+}$(the meson $\tilde{D}_{s 1}$ ) is possible. Comparison of the features of the four possible classifications for $D_{s J}(3040)$ shows that, due to the large mass, several decay modes are possible: to a member of the fundamental heavy doublet plus a light pseudoscalar or vector meson and to a member of an excited heavy doublet and a light pseudoscalar meson [21]. In the heavy quark limit, the two $J^{P}=1^{+}$are expected to be broader than the two $J^{P}=2^{+}$states, hence $D_{s J}(3040)$ is likely to be identified with one of the two axial-vector mesons. A distinction between the two is provided by the $D K^{*}$ and $D_{s} \phi$ decay modes, since the widths to these final states are larger for $\tilde{D}_{s 1}^{\prime}$ than for $\tilde{D}_{s 1}$. This justifies the classification of $D_{s J}(3040)$ as one of the two states with $J^{P}=1^{+}, n=2$, proposed in Table [ The features of the corresponding spin and non strange partners can be predicted accordingly [21].

The last four states in Table \are the non-strange $c \bar{q}$ mesons discovered by the BaBar collaboration in the process $e^{+} e^{-} \rightarrow c \bar{c} \rightarrow D^{(*)} \pi X[22]$. The four new resonances are found with decay modes:

- $D^{0}(2550)$ to $D^{*+} \pi^{-}$;

- $D^{* 0}(2600)$ to $D^{+} \pi^{-}$and $D^{*+} \pi^{-}$, and the isospin partner $D^{*+}(2600)$ to $D^{0} \pi^{+}$;

- $D^{* 0}(2760)$ to $D^{+} \pi^{-}$, and the isospin partner $D^{*+}(2760)$ to $D^{0} \pi^{+}$;

- $D^{* 0}(2750)$ to $D^{*+} \pi^{-}$.

Table II: Measured mass and width of the observed excited mesons with open charm. All the results are from the PDG 7], excluding the data concerning $D^{* 0,+}(2600), D^{0}(2750)$ and $D^{* 0,+}(2760)$ which are BaBar measurements [22]; the widths of $D^{*+}(2600)$ and $D^{*+}(2600)$ are kept fixed in the experimental analysis [22]. The quoted bounds are at 95\% CL.

\begin{tabular}{|ccc|ccc|}
\hline$c \bar{q}$ & mass $(\mathrm{MeV})$ & $\Gamma(\mathrm{MeV})$ & $c \bar{s}$ & mass $(\mathrm{MeV})$ & $\Gamma(\mathrm{MeV})$ \\
\hline$D_{0}^{* 0}(2400)$ & $2318 \pm 29$ & $267 \pm 40$ & & & \\
\hline$D_{0}^{* \pm}(2400)$ & $2403 \pm 14 \pm 35$ & $283 \pm 24 \pm 34$ & $D_{s 0}^{*}(2317)$ & $2317.8 \pm 0.6$ & $<3.8$ \\
\hline$D_{1}^{\prime 0}(2430)$ & $2427 \pm 26 \pm 25$ & $384 \pm_{75}^{107} \pm 74$ & & & \\
\hline \multicolumn{7}{c}{} & & $D_{s 1}^{\prime}(2460)$ & $2459.6 \pm 0.6$ & $<3.5$ \\
\hline \hline$D_{1}^{0}(2420)$ & $2421.3 \pm 0.6$ & $27.1 \pm 2.7$ & & & \\
\hline$D_{1}^{ \pm}(2420)$ & $2423.4 \pm 3.1$ & $26 \pm 6$ & $D_{s 1}(2536)$ & $2535.12 \pm 0.13$ & $0.92 \pm 0.03 \pm 0.04$ \\
\hline$D_{2}^{* 0}(2460)$ & $2462.6 \pm 0.7$ & $49.0 \pm 1.4$ & & & \\
\hline$D_{2}^{* \pm}(2460)$ & $2464.4 \pm 1.9$ & $37 \pm 6$ & $D_{s 2}^{*}(2573)$ & $2572.6 \pm 0.9$ & $20 \pm 5$ \\
\hline \hline$D^{0}(2550)$ & $2539.4 \pm 4.5 \pm 6.8$ & $130 \pm 12 \pm 13$ & & & \\
\hline$D^{* 0}(2600)$ & $2608.7 \pm 2.4 \pm 2.5$ & $93 \pm 6 \pm 13$ & & & \\
\hline$D^{*+}(2600)$ & $2621.3 \pm 3.7 \pm 4.2$ & 93 (fixed) & $D_{s 1}^{*}(2700)$ & $2709 \pm_{6}^{9}$ & $125 \pm 30$ \\
\hline \hline$D^{0}(2750)$ & $2752.4 \pm 1.7 \pm 2.7$ & $71 \pm 6 \pm 11$ & & & \\
\hline$D^{* 0}(2760)$ & $2763.3 \pm 2.3 \pm 2.3$ & $60.9 \pm 5.1 \pm 3.6$ & \multicolumn{3}{|c|}{} \\
\hline$D^{*+}(2760)$ & $2769.7 \pm 3.8 \pm 1.5$ & 60.9 (fixed) & $D_{s J}(2860)$ & $2862 \pm 2 \pm_{2}^{5}$ & $48 \pm 3 \pm 6$ \\
\hline \hline & & $D_{s J}(3040)$ & $3044 \pm 8 \pm_{5}^{30}$ & $239 \pm 35 \pm 42$ \\
\hline
\end{tabular}


Their measured mass and width are reported in Table II The BaBar collaboration has also measured the ratios

$$
\begin{aligned}
& \frac{B R\left(D^{* 0}(2600) \rightarrow D^{+} \pi^{-}\right)}{B R\left(D^{* 0}(2600) \rightarrow D^{*+} \pi^{-}\right)}=0.32 \pm 0.02 \pm 0.09 \\
& \frac{B R\left(D^{* 0}(2760) \rightarrow D^{+} \pi^{-}\right)}{B R\left(D^{* 0}(2750) \rightarrow D^{*+} \pi^{-}\right)}=0.42 \pm 0.05 \pm 0.11 .
\end{aligned}
$$

In the case of the final state $D^{*+} \pi^{-}$, important piece of information comes from the distribution in $\cos \theta_{H}$, with $\theta_{H}$ the helicity angle between the primary pion $\pi^{-}$and the slow pion $\pi^{+}$from the $D^{*+}$ decay. The measured $\cos \theta_{H}$ distribution for $D^{*}(2600)$ is consistent with the assignment of natural parity to this state, which also agrees with the observation in both $D \pi$ and $D^{*} \pi$; moreover, the angular distribution for $D^{0}(2550)$ behaves like $\sim \cos ^{2} \theta_{H}$, as expected for a $J^{P}=0^{-}$state.

On the basis of these observations, the Babar collaboration suggested that $\left(D(2550), D^{*}(2600)\right)$ compose the $\tilde{H}$, $J^{P}=\left(0^{-}, 1^{-}\right)$doublet of $n=2$ radial excitations of $\left(D, D^{*}\right)$ mesons, while $\left(D(2750), D^{*}(2760)\right)$, can be identified with the $\ell=2, n=1$ states [22], mainly from comparison of the measured masses with quark model results [23]. Since there are two possible doublets with $\ell=2$, the identification with the $J^{P}=\left(2^{-}, 3^{-}\right)$doublet would come together with the hypothesis $D_{s J}(2860)=D_{s 3}$, and in this case $D_{s J}(2860)$ and $D^{*}(2760)$ would be corresponding states with and without strangeness. We have classified the four new states in Table I according to these conclusions. However, we have mentioned that another possibility is $D_{s J}(2860)$ being identified with the $\tilde{D}_{s 2}^{*}$ meson. In such a case, if $D^{*}(2760)$ is viewed as the non strange partner of $D_{s J}(2860)$, it can be the state $\tilde{D}_{2}^{*}$, and $D(2750)$ its spin partner $\tilde{D}_{1}^{\prime}$, both filling the doublet $\tilde{T}$. In the quark model, the masses of the states in the $\left(D_{2}^{\prime *}, D_{3}\right)$ doublet are found to be $(2.775 \mathrm{GeV}, 2.799 \mathrm{GeV})$, while in the case of the $n=2\left(\tilde{D}_{1}^{\prime} D_{2}^{*}\right)$ doublet the obtained masses are $(2.995 \mathrm{GeV}, 3.035$ $\mathrm{GeV}$ ) [20], findings that also support the first one of these two possible assignments.

Let us turn to the beauty sector. As in the case of charm, the $J^{P}=\left(0^{-}, 1^{-}\right)$lightest doublet is well established and is included in Table \ together with the other observed resonances. The measured masses and widths of the excited states are reported in Table III. First observations of open beauty resonances were gained by the LEP collaborations [24, 25], using inclusive or semi-exclusive $B$ decays which made impossible the separation of the states. More recently, the CDF and D0 collaborations at the Tevatron reported evidence of non strange excited beauty mesons, which can be identified with the $\left(B_{1}, B_{2}^{*}\right)$ components of the $s_{\ell}^{P}=\frac{3}{2}^{+}$doublet. They are found in the decays $B_{2}^{* 0} \rightarrow B^{+} \pi^{-}, B^{*+} \pi^{-}$ and $B_{1}^{0} \rightarrow B^{*+} \pi^{-}[26,27]$. Analogously, for strange-beauty mesons the first observation of $p$-wave states was not able to separate the individual components [24]. Recent CDF and D0 studies have reported evidence of $B_{s 1}$ decaying to $B^{*+} K^{-}$, and of $B_{s 2}^{*}$ decaying to $B^{+} K^{-}[28,29]$. These resonances can be assigned to the $s_{\ell}^{P}=\frac{3}{2}^{+}$beauty doublet with strangeness. Confirmation of these orbitally excited $B$ and $B_{s}$ mesons, with compatible masses and widths, has been obtained by the LHCb collaboration [30].

Table III: Measured mass and width of the observed open beauty excited mesons [7].

\begin{tabular}{|ccc|ccc|}
\hline$b \bar{q}$ & mass $(\mathrm{MeV})$ & $\Gamma(\mathrm{MeV})$ & $b \bar{s}$ & $\operatorname{mass}(\mathrm{MeV})$ & $\Gamma(\mathrm{MeV})$ \\
\hline$B_{1}^{0}(5721)$ & $5723.4 \pm 2.0$ & & $B_{s 1}^{0}(5830)$ & $5829.4 \pm 0.7$ & \\
\hline$B_{2}^{* 0}(5747)$ & $5743 \pm 5$ & $22.7 \pm_{3.2}^{3.8} \pm_{10.2}^{3.2}$ & $B_{s 2}^{* 0}(5840)$ & $5839.7 \pm 0.6$ & \\
\hline
\end{tabular}

\section{MESON MASS PARAMETERS AND PREDICTIONS}

The HQ symmetries and the mass measurements permit to predict the masses of not yet observed open charm and open beauty resonances, filling a few empty spaces in Table [. The preliminary step is to determine the average masses $\bar{M}_{F}$ in Eqs.(77), the mass splittings $\Delta_{F}$ between doublets and the splittings $\lambda_{F}$ between spin partners in a doublet defined in Eqs. (9) for all the observed states in Table I The results are collected in Table IV] Flavour symmetry implies that the mass splittings $\Delta_{F}$ between doublets are the same regardless of the heavy quark flavour. Furthermore, also the mass splitting $\lambda_{F}$ between spin partners in a doublet should not depend on the heavy flavour. Considering the various entries in Table IV] we see that these statements are experimentally violated both by light flavour and heavy quark mass effects, since such entries incorporate higher order symmetry breaking terms: in particular, the strange quark mass effect is clearly visible in the average mass parameters $\bar{M}_{F}$.

Using this input, we can elaborate several predictions for the masses of unobserved states. Let us consider $\tilde{D}_{s}$, the spin partner of $\tilde{D}_{s}^{*}$ which we have identified with $D_{s 1}^{*}(2700)$. If the effect of the strange quark is to shift the mass of a given state by the same amount in the fundamental and in the $n=2$ radial excitation doublet, we have 
$M_{D_{s}}-M_{D^{0}}=M_{\tilde{D}_{s}}-M_{\tilde{D}^{0}}$. Identifying $\tilde{D}^{0}$ with the observed $D^{0}(2550)$, we can infer that $M_{\tilde{D}_{s}}=2643 \pm 8 \mathrm{MeV}$. The consistency of the identification of $D^{* 0}(2600)$ with the spin partner of $D^{0}(2550)$, i.e. with the state $\tilde{D}^{* 0}$, can analogously be checked. From $M_{D_{s}^{*}}-M_{D^{* 0}}=M_{\tilde{D}_{s}^{*}}-M_{\tilde{D}^{* 0}}$ we obtain: $M_{\tilde{D}^{* 0}}=2604 \pm 9 \mathrm{MeV}$, which supports the identification of $D^{* 0}(2600)$ with $\tilde{D}^{* 0}$. A compatible result is obtained using the $\lambda_{\tilde{H}}$ parameter in Table IV to predict the $\tilde{D}_{s}$ mass: $M_{\tilde{D}_{s}}=2643 \pm 13 \mathrm{MeV}$. This allows us to conclude that the masses of all $n=1$ and $2, J_{s_{\ell}}^{P}=\left(0^{-}, 1^{-}\right)_{1 / 2}$ charmed mesons with and without strangeness, are determined, see Table $\nabla$

In the case of the doublet $J_{s_{\ell}}^{P}=\left(2^{-}, 3^{-}\right)_{5 / 2}$, if the two states $D(2750)$ and $D^{*}(2760)$ are the $\left(D_{2}^{\prime *}, D_{3}\right)$ members of this $\mathrm{X}^{\prime}, c \bar{q}$ doublet, we can predict the mass of the spin partner $D_{s 2}^{\prime *}$ of $D_{s J}(2860)$ identified with $D_{s 3}$. From $M_{D_{s 2}^{\prime *}}=M_{D_{s 3}}-\left(M_{D_{3}}-M_{D_{2}^{\prime *}}\right)$ we have: $M_{D_{s 2}^{\prime *}}=2851 \pm 7 \mathrm{MeV}$. This prediction for the mass of the spin partner of $D_{s J}(2860)$ holds independently of the identification of the latter, it only relies on the assumption that $D_{s J}(2860)$ and $D^{*}(2760)$ have the same quantum numbers and differ for the strangeness.

Using the estimated masses of $\tilde{D}_{s}$ and $D_{s 2}^{\prime *}$ we obtain: $\bar{M}_{\tilde{H}}=2692.5 \pm 7.0 \mathrm{MeV}, \Delta_{\tilde{H}}=616 \pm 7 \mathrm{MeV}, \lambda_{\tilde{H}}=$ $(210 \pm 19 \mathrm{MeV})^{2}$, and $\bar{M}_{X^{\prime}}=2857.5 \pm 4.3 \mathrm{MeV}, \Delta_{X^{\prime}}=781 \pm 4 \mathrm{MeV}, \lambda_{X^{\prime}}=(114 \pm 46 \mathrm{MeV})^{2}$ for $c \bar{s}$ mesons. Another possibility is that $D_{s J}(2860)$ and $\left(D(2750), D^{*}(2760)\right)$ belong to the $n=2, \tilde{T}$ doublets: In this case we would get $\bar{M}_{\tilde{T}}=2759.2 \pm 2.4 \mathrm{MeV}, \Delta_{\tilde{T}}=787.8 \pm 2.4 \mathrm{MeV}$ and $\lambda_{\tilde{T}}=(106 \pm 22 \mathrm{MeV})^{2}$.

In the HQ limit, charm data can be exploited to make predictions for beauty. The procedure we adopt, based on the Lagrangian (3), (8), is to assume the equalities

$$
\begin{aligned}
\Delta_{F}^{(c)} & =\Delta_{F}^{(b)} \\
\lambda_{F}^{(c)} & =\lambda_{F}^{(b)}
\end{aligned}
$$

for $F=\tilde{H}, S, T, X^{\prime}$ and $\tilde{T}$, and to use these two expressions, with the l.h.s. experimentally determined, to predict the masses of the two states in the corresponding beauty doublets. The results are collected in Table VI. If $D_{s J}(2860)$ and $\left.\left(D(2750), D^{*}(2760)\right)\right)$ are assigned to the $n=2, \tilde{T}$ doublets, the last two columns in this Table would represent the predictions for $\tilde{B}_{(s) 1}^{\prime}$ and $\tilde{B}_{(s) 2}$, respectively. It is worth remarking that the masses of $B_{s 0}^{*}$ and $B_{s 1}^{\prime}$ are below the $B K$ and $B^{*} K$ thresholds, and therefore these two mesons are expected to be very narrow, with main decays into $B_{s} \pi^{0}$ and $B_{s}^{*} \pi^{0}$ [31 33].

Table IV: Values of the spin averaged masses $\bar{M}_{F}$ (in $\mathrm{MeV}$ ), of the mass splittings $\Delta_{F}$ (in $\mathrm{MeV}$ ) and of the hyperfine splitting parameters $\lambda_{F}\left(\right.$ in $\left.\mathrm{MeV}^{2}\right)$ defined in Eqs.(77), (9).

\begin{tabular}{|lcccccc|}
\hline & $c \bar{u}$ & $c \bar{d}$ & $c \bar{s}$ & $b \bar{u}$ & $b \bar{d}$ & $b \bar{s}$ \\
\hline $\bar{M}_{H}$ & $1971.45 \pm 0.12$ & $1975.12 \pm 0.10$ & $2076.4 \pm 0.4$ & $5313.7 \pm 0.3$ & $5313.8 \pm 0.3$ & $5403 \pm 2$ \\
$\bar{M}_{\tilde{H}}$ & $2591.4 \pm 3.3$ & & & & \\
$\bar{M}_{S}$ & $2400 \pm 28$ & & $2424.1 \pm 0.5$ & & & \\
$\bar{M}_{T}$ & $2447.1 \pm 0.5$ & $2449.0 \pm 1.6$ & $2558.6 \pm 0.6$ & & & \\
$\bar{M}_{X^{\prime}}$ & $2758.8 \pm 2.3$ & & & & \\
\hline$\Delta_{S}$ & $429 \pm 28$ & & $347.7 \pm 0.6$ & & & \\
$\Delta_{T}$ & $475.7 \pm 0.5$ & $473.9 \pm 1.6$ & $482.2 \pm 0.7$ & & & \\
$\Delta_{X^{\prime}}$ & $787.4 \pm 2.3$ & & & & \\
\hline$\lambda_{H}$ & $(262.3 \pm 0.2)^{2}$ & $(261.2 \pm 0.2)^{2}$ & $(270.9 \pm 0.6)^{2}$ & $(246.8 \pm 1.2)^{2}$ & $(245.9 \pm 1.2)^{2}$ & $(256.3 \pm 6.4$ \\
$\lambda_{\tilde{H}}$ & $(211.2 \pm 13.4)^{2}$ & & & & \\
$\lambda_{S}$ & $(254 \pm 54)^{2}$ & & $(290.9 \pm 0.9)^{2}$ & & \\
$\lambda_{T}$ & $(195 \pm 2)^{2}$ & $(193 \pm 7)^{2}$ & $(189.2 \pm 2.1)^{2}$ & & & \\
$\lambda_{X^{\prime}}$ & $(112 \pm 24)^{2}$ & & & & & \\
\hline
\end{tabular}


Table V: Predicted mass and width (in $\mathrm{MeV}$ ) of two not yet observed excited charm mesons, quoted together with the other members of their respective doublets.

\begin{tabular}{|cc|cc|cc|}
\hline & & $\tilde{D}_{(s)}\left(0^{-}, n=2\right)$ & $\tilde{D}_{(s)}^{*}\left(1^{-}, n=2\right)$ & $D_{(s) 2}^{* *}\left(2^{-}\right)$ & $D_{(s) 3}\left(3^{-}\right)$ \\
\hline$c \bar{q}$ & & $D(2550)$ & $D^{*}(2600)$ & $D(2750)$ & $D(2760)$ \\
$c \bar{s}$ & mass & $2643 \pm 13$ & $D_{s 1}^{*}(2700)$ & $2851 \pm 7$ & $D_{s J}(2860)$ \\
& $\Gamma$ & $33.5 \pm 3.3$ & & $20.5 \pm 2.4$ & \\
\hline
\end{tabular}

Table VI: Predicted mass and width (in $\mathrm{MeV}$ ) of doublets of excited beauty mesons. For the decay widths of $B_{s 0}^{*}$ and $B_{s 1}^{\prime}$ see the text.

\begin{tabular}{|cc|cc|cc|cc|}
\hline & & $\tilde{B}_{(s)}\left(0^{-}, n=2\right)$ & $\tilde{B}_{(s)}^{*}\left(1^{-}, n=2\right)$ & $B_{(s) 0}^{*}\left(0^{+}\right)$ & $B_{(s) 1}^{\prime}\left(1^{+}\right)$ & $B_{(s) 2}^{\prime *}\left(2^{-}\right)$ & $B_{(s) 3}\left(3^{-}\right)$ \\
\hline$b \bar{q}$ & mass & $5911.1 \pm 4.9$ & $5941.2 \pm 3.2$ & $5708.2 \pm 22.5$ & $5753.3 \pm 31.1$ & $6098.2 \pm 2.4$ & $6103.1 \pm 2.6$ \\
& $\Gamma$ & $149 \pm 15$ & $186 \pm 18$ & $269 \pm 58$ & $268 \pm 70$ & $103 \pm 8$ & $129 \pm 10$ \\
& & & & & & \\
$b \bar{s}$ & mass & $5997.3 \pm 6.1$ & $6026.6 \pm 7.9$ & $5706.6 \pm 1.2$ & $5765.6 \pm 1.2$ & $6181.3 \pm 5.2$ & $6186.3 \pm 4.6$ \\
& $\Gamma$ & $76 \pm 9$ & $118 \pm 14$ & & & $57 \pm 6$ & $78.4 \pm 7.3$ \\
\hline
\end{tabular}

\section{STRONG TWO-BODY DECAYS TO H AND A LIGHT PSEUDOSCALAR MESON}

To describe the decays $F \rightarrow H M$, with $F=H, S, T, X, X^{\prime}$ and $M$ a light pseudoscalar meson, at the leading order in the light meson momentum and heavy quark mass expansion, we can employ the Lagrangian interaction terms [4]:

$$
\begin{aligned}
\mathcal{L}_{H} & =g \operatorname{Tr}\left[\bar{H}_{a} H_{b} \gamma_{\mu} \gamma_{5} \mathcal{A}_{b a}^{\mu}\right] \\
\mathcal{L}_{S} & =h \operatorname{Tr}\left[\bar{H}_{a} S_{b} \gamma_{\mu} \gamma_{5} \mathcal{A}_{b a}^{\mu}\right]+h . c . \\
\mathcal{L}_{T} & =\frac{h^{\prime}}{\Lambda_{\chi}} \operatorname{Tr}\left[\bar{H}_{a} T_{b}^{\mu}\left(i D_{\mu} \mathcal{A}+i \not \mathcal{A}_{\mu}\right)_{b a} \gamma_{5}\right]+h . c . \\
\mathcal{L}_{X} & =\frac{k^{\prime}}{\Lambda_{\chi}} \operatorname{Tr}\left[\bar{H}_{a} X_{b}^{\mu}\left(i D_{\mu} \mathcal{A}+i \not D \mathcal{A}_{\mu}\right)_{b a} \gamma_{5}\right]+h . c . \\
\mathcal{L}_{X^{\prime}} & =\frac{1}{\Lambda_{\chi}^{2}} \operatorname{Tr}\left[\bar{H}_{a} X_{b}^{\prime \mu \nu}\left[k_{1}\left\{D_{\mu}, D_{\nu}\right\} \mathcal{A}_{\lambda}+k_{2}\left(D_{\mu} D_{\lambda} \mathcal{A}_{\nu}+D_{\nu} D_{\lambda} \mathcal{A}_{\mu}\right)\right]_{b a} \gamma^{\lambda} \gamma_{5}\right]+\text { h.c. }
\end{aligned}
$$

The chiral symmetry-breaking scale $\Lambda_{\chi}$ is set to $\Lambda_{\chi}=1 \mathrm{GeV} . \mathcal{L}_{S}$ and $\mathcal{L}_{T}$ describe transitions of positive parity heavy mesons with the emission of light pseudoscalar mesons in $s-$ and $d$ - wave, respectively, and $g, h$ and $h^{\prime}$ are effective coupling constants. $\mathcal{L}_{X}$ and $\mathcal{L}_{X^{\prime}}$ describe the transitions of higher mass mesons of negative parity, belonging to the $X$ and $X^{\prime}$ doublets, with the emission of light pseudoscalar mesons in $p-$ and $f$ - wave, with coupling constants $k^{\prime}$, $k_{1}$ and $k_{2}$ (we set $k=k_{1}+k_{2}$ ). At the same order in the expansion in the light meson momentum, the structure of the Lagrangian terms for radial excitations of the various doublets does not change since it is only dictated by the spin-flavour and chiral symmetries, but the coupling constants must be replaced by new ones denoted by $\tilde{g}, \tilde{h}$, and so on. This formulation is useful since meson transitions into final states obtained by flavour and heavy quark spin trasnformations can be related in a straightforward way. The expressions of the decay widths obtained from Eqs.(13), considering the various doublets which the decaying meson belongs to, are the following:

- Decaying meson $H=\left(P, P^{*}\right)$ or $\tilde{H}=\left(\tilde{P}, \tilde{P}^{*}\right)$ :

$$
\begin{aligned}
\Gamma\left(P^{*} \rightarrow P M\right) & =C_{M} \frac{g^{2}}{6 \pi f_{\pi}^{2}} \frac{M_{P}}{M_{P^{*}}}\left|\vec{p}_{M}\right|^{3} \\
\Gamma\left(\tilde{P}^{*} \rightarrow P M\right) & =C_{M} \frac{\tilde{g}^{2}}{6 \pi f_{\pi}^{2}} \frac{M_{P}}{M_{\tilde{P}^{*}}}\left|\vec{p}_{M}\right|^{3} \\
\Gamma\left(\tilde{P}_{i}^{*} \rightarrow P_{f}^{*} M\right) & =C_{M} \frac{\tilde{g}^{2}}{3 \pi f_{\pi}^{2}} \frac{M_{P_{f}^{*}}}{M_{\tilde{P}_{i}^{*}}}\left|\vec{p}_{M}\right|^{3} \\
\Gamma\left(\tilde{P} \rightarrow P^{*} M\right) & =C_{M} \frac{\tilde{g}^{2}}{2 \pi f_{\pi}^{2}} \frac{M_{P^{*}}}{M_{\tilde{P}}}\left|\vec{p}_{M}\right|^{3} .
\end{aligned}
$$


- Decaying $S=\left(P_{0}^{*}, P_{1}^{\prime}\right)$ :

$$
\begin{aligned}
\Gamma\left(P_{0}^{*} \rightarrow P M\right) & =C_{M} \frac{h^{2}}{2 \pi f_{\pi}^{2}} \frac{M_{P}}{M_{P_{0}^{*}}}\left[m_{M}^{2}+\left|\vec{p}_{M}\right|^{2}\right]\left|\vec{p}_{M}\right| \\
\Gamma\left(P_{1}^{\prime} \rightarrow P^{*} M\right) & =C_{M} \frac{h^{2}}{2 \pi f_{\pi}^{2}} \frac{M_{P^{*}}}{M_{P_{1}^{\prime}}}\left[m_{M}^{2}+\left|\vec{p}_{M}\right|^{2}\right]\left|\vec{p}_{M}\right| .
\end{aligned}
$$

- Decaying $T=\left(P_{1}, P_{2}^{*}\right)$ or $\tilde{T}=\left(\tilde{P}_{1}, \tilde{P}_{2}^{*}\right)$ :

$$
\begin{aligned}
\Gamma\left(P_{1} \rightarrow P^{*} M\right) & =C_{M} \frac{2 h^{\prime 2}}{3 \pi f_{\pi}^{2}} \frac{M_{P^{*}}}{M_{P_{1}}}\left|\vec{p}_{M}\right|^{5} \\
\Gamma\left(P_{2}^{*} \rightarrow P M\right) & =C_{M} \frac{4 h^{\prime 2}}{15 \pi f_{\pi}^{2}} \frac{M_{P}}{M_{P_{2}^{*}}}\left|\vec{p}_{M}\right|^{5} \\
\Gamma\left(P_{2}^{*} \rightarrow P^{*} M\right) & =C_{M} \frac{2 h^{\prime 2}}{5 \pi f_{\pi}^{2}} \frac{M_{P^{*}}}{M_{P_{2}^{*}}}\left|\vec{p}_{M}\right|^{5} .
\end{aligned}
$$

- Decaying $X=\left(P_{1}^{*}, P_{2}\right)$ :

$$
\begin{gathered}
\Gamma\left(P_{1}^{*} \rightarrow P M\right)=C_{M} \frac{4 k^{\prime 2}}{9 \pi f_{\pi}^{2}} \frac{M_{P}}{M_{P_{1}^{*}}}\left[m_{M}^{2}+\left|\vec{p}_{M}\right|^{2}\right]\left|\vec{p}_{M}\right|^{3} \\
\Gamma\left(P_{1}^{*} \rightarrow P^{*} M\right)=C_{M} \frac{2 k^{\prime 2}}{9 \pi f_{\pi}^{2}} \frac{M_{P^{*}}}{M_{P_{1}^{*}}}\left[m_{M}^{2}+\left|\vec{p}_{M}\right|^{2}\right]\left|\vec{p}_{M}\right|^{3} \\
\Gamma\left(P_{2} \rightarrow P^{*} M\right)=C_{M} \frac{2 k^{\prime 2}}{3 \pi f_{\pi}^{2}} \frac{M_{P^{*}}}{M_{P_{2}}}\left[m_{M}^{2}+\left|\vec{p}_{M}\right|^{2}\right]\left|\vec{p}_{M}\right|^{3} .
\end{gathered}
$$

- Decaying $X^{\prime}=\left(P_{2}^{\prime *}, P_{3}\right)$ :

$$
\begin{aligned}
\Gamma\left(P_{2}^{\prime *} \rightarrow P^{*} M\right) & =C_{M} \frac{4 k^{2}}{15 \pi f_{\pi}^{2}} \frac{M_{P^{*}}}{M_{P_{2}^{\prime *}}}\left|\vec{p}_{M}\right|^{7} \\
\Gamma\left(P_{3} \rightarrow P M\right) & =C_{M} \frac{4 k^{2}}{35 \pi f_{\pi}^{2}} \frac{M_{P}}{M_{P_{3}}}\left|\vec{p}_{M}\right|^{7} \\
\Gamma\left(P_{3} \rightarrow P^{*} M\right) & =C_{M} \frac{16 k^{2}}{105 \pi f_{\pi}^{2}} \frac{M_{P^{*}}}{M_{P_{3}}}\left|\vec{p}_{M}\right|^{7} .
\end{aligned}
$$

The coefficients $C_{M}$ are different for the various light pseudoscalar mesons: $C_{\pi^{+}}=C_{K^{+}}=1, C_{\pi^{0}}=C_{K_{S}}=\frac{1}{2}$, $C_{\eta}=\frac{2}{3} \cdot \vec{p}_{M}$ is the three momentum of $M$. Notice that only for the states $\tilde{P}^{*}$ in $\tilde{H}, P_{2}^{*}$ in $T, P_{1}^{*}$ in $X$ and $P_{3}$ in $X^{\prime}$, both the decays to $P M$ and $P^{*} M$ are allowed, while the other resonances can decay either to $P M$ or to $P^{*} M$, a useful observation for the classification of the states.

The decay rates depend on effective coupling constants which need to be specified. Model dependence in a determination of such couplings can be avoided considering ratios of widths in which the constants cancel out. Therefore, for each meson $F_{(s)}$ that can decay both to $P_{(s)} M$ and $P_{(s)}^{*} M$, we focus on the following ratios, considering as reference modes the decay to $D \pi$ for the non strange mesons, and to $D K$ for the strange ones:

$$
\begin{aligned}
R_{\pi}^{(F)} & =\frac{B R\left(F \rightarrow D^{*} \pi\right)}{B R(F \rightarrow D \pi)}, \\
R_{K}^{\left(F_{s}\right)} & =\frac{B R\left(F_{s} \rightarrow D^{*} K\right)}{B R\left(F_{s} \rightarrow D K\right)}, \quad R_{\eta}^{\left(F_{s}\right)}=\frac{B R\left(F_{s} \rightarrow D_{s} \eta\right)}{B R\left(F_{s} \rightarrow D K\right)}, \quad R_{\eta}^{*\left(F_{s}\right)}=\frac{B R\left(F_{s} \rightarrow D_{s}^{*} \eta\right)}{B R\left(F_{s} \rightarrow D K\right)} .
\end{aligned}
$$

$D^{(*)} \pi(K)$ indicates $D^{(*) 0} \pi^{+}\left(K^{+}\right)+D^{(*)+} \pi^{0}\left(K_{S}\right)$ for charged states, and $D^{(*) 0} \pi^{0}\left(K_{S}\right)+D^{(*)+} \pi^{-}\left(K^{-}\right)$for neutral ones. Such ratios can be experimentally determined, so a comparison with the theoretical outcome is possible.

In Table VII we collect the predictions for the charmed states $D^{*}(2600)$ and $D_{s 1}^{*}(2700)$, identified with $\tilde{D}^{*}$ and $\tilde{D}_{s}^{*}$, respectively; for $D_{2}^{* 0}(2460)$ and $D_{s 2}^{*}(2573)$ and for $D^{*}(2760)$ and $D_{s J}(2860)$ identified with the states $D_{3}$ and $D_{s 3}$, as assumed in Table 1 The case of $D_{(s) 1}^{*}$ in the doublet $X$ is not included in the Table since there are at present no 
Table VII: Theoretical ratios $R_{M}^{(F)}$ for charmed and beauty mesons. The results are obtained identifying $D^{* 0}(2760)$ as $D_{3}$ and $D_{s J}(2860)$ as $D_{s 3}$.

\begin{tabular}{|c|c||c|ccc|}
\hline$c \bar{q}$ & $R_{\pi}$ & $c \bar{s}$ & $R_{K^{0}}$ & $R_{\eta}$ & $R_{\eta}^{*}$ \\
\hline$D^{* 0}(2600)$ & $1.22 \pm 0.01$ & $D_{s 1}^{*}(2700)$ & $0.91 \pm 0.03$ & $0.195 \pm 0.006$ & $0.05 \pm 0.01$ \\
$D_{2}^{* 0}(2460)$ & $0.440 \pm 0.001$ & $D_{s 2}^{*}(2573)$ & $0.086 \pm 0.002$ & $0.018 \pm 0.001$ & - \\
$D^{* 0}(2760)$ & $0.514 \pm 0.004$ & $D_{s J}(2860)$ & $0.39 \pm 0.01$ & $0.132 \pm 0.003$ & $0.025 \pm 0.001$ \\
\hline \hline$b \bar{q}$ & $R_{\pi}$ & $b \bar{s}$ & $R_{K}$ & $R_{\eta}$ & $R_{\eta}^{*}$ \\
\hline$\tilde{B}^{*}$ & $1.63 \pm 0.005$ & $\tilde{B}_{s}^{*}$ & $1.43 \pm 0.015$ & $0.132 \pm 0.008$ & $0.11 \pm 0.015$ \\
$B_{2}^{*}$ & $0.87 \pm 0.01$ & $B_{s 2}^{*}$ & $0.07 \pm 0.005$ & - & - \\
$B_{3}$ & $0.92 \pm 0.005$ & $B_{s 3}$ & $0.815 \pm 0.006$ & $0.103 \pm 0.002$ & $0.063 \pm 0.003$ \\
\hline
\end{tabular}

candidates for it; however, we consider it in the following. In the alternative classification of $D^{* 0}(2760)$ as $\tilde{D}_{2}^{*}$ we obtain $R_{\pi}=0.775 \pm 0.003$; analogously, identifying $D_{s J}(2860)$ with $\tilde{D}_{s 2}^{*}$ we get $R_{K}=0.63 \pm 0.01, R_{\eta}=0.19 \pm 0.002$ and $R_{\eta}^{*}=0.07 \pm 0.003$.

For some of the resonances considered in Table VII experimental data are available. For $D_{2}^{*}(2460)$ one has [7]:

$$
\begin{aligned}
\frac{\Gamma_{1}}{\Gamma_{2}} & =\frac{\Gamma\left(D_{2}^{*}(2460)^{ \pm} \rightarrow D^{0} \pi^{+}\right)}{\Gamma\left(D_{2}^{*}(2460)^{ \pm} \rightarrow D^{* 0} \pi^{+}\right)}=1.9 \pm 1.1 \pm 0.3 \\
R_{12} & =\frac{\Gamma_{1}}{\Gamma_{1}+\Gamma_{2}}=0.62 \pm 0.03 \pm 0.02
\end{aligned}
$$

for the charged state, and

$$
\begin{aligned}
\frac{\Gamma_{1}}{\Gamma_{2}} & =\frac{\Gamma\left(D_{2}^{*}(2460)^{0} \rightarrow D^{+} \pi^{-}\right)}{\Gamma\left(D_{2}^{*}(2460)^{0} \rightarrow D^{*+} \pi^{-}\right)}=1.56 \pm 0.16 \pm 0.3 \\
R_{12} & =\frac{\Gamma_{1}}{\Gamma_{1}+\Gamma_{2}}=0.62 \pm 0.03 \pm 0.02
\end{aligned}
$$

for the neutral one. For these quantities the theoretical results are

$$
\begin{array}{ll}
\left(\frac{\Gamma_{1}}{\Gamma_{2}}\right)_{\text {charged }}=2.266 \pm 0.015 & \left(R_{12}\right)_{\text {charged }}=0.694 \pm 0.001 \\
\left(\frac{\Gamma_{1}}{\Gamma_{2}}\right)_{\text {neutral }}=2.280 \pm 0.007 & \left(R_{12}\right)_{\text {neutral }}=0.695 \pm 0.001 .
\end{array}
$$

The comparison of predictions with data shows a deviation from the heavy quark limit in the case of the neutral channel. However, the PDG result is obtained averaging several data in the range $\left(\frac{\Gamma_{1}}{\Gamma_{2}}\right)_{\text {neutral }} \in[2.2-3.0]$, and the average is dominated by a single measurement provided by BaBar collaboration [22], which requires a confirmation.

The corresponding charmed-strange resonance is $D_{s 2}^{*}(2573)$, identified as the $2^{+}$member of the $\ell=1, s_{\ell}^{P}=\frac{3}{2}$, $T$ doublet. It decays to $D^{(*)+} K_{S}, D^{(*) 0} K^{+}, D_{s} \eta$, and is below the $D_{s}^{*} \eta$ threshold. There are no experimental data for the ratios in Table VII however, the PDG quotes the upper bound: $\frac{B R\left(D_{s 2}^{*}(2573) \rightarrow D^{* 0} K^{+}\right)}{B R\left(D_{s 2}^{*}(2573) \rightarrow D^{0} K^{+}\right)}<0.33$, which is satisfied by our result $\frac{B R\left(D_{s 2}^{*}(2573) \rightarrow D^{* 0} K^{+}\right)}{B R\left(D_{s 2}^{*}(2573) \rightarrow D^{0} K^{+}\right)}=0.091 \pm 0.002$.

The $D_{s 1}^{*}(2700)$ has been treated in Ref.[18], where the ratios in Table VII were analyzed. The BaBar measurement of the first ratio in Eq.(10) [17] is in agreement with the theoretical outcome reported in the Table, and this supports the classification of this state as $\tilde{D}_{s 1}^{*}$.

Let us turn to the first radial excitation of $D^{*}$, the $\tilde{D}^{*}$. As discussed in the previous Sections, there are hints that the observed $D^{*}(2600)$ might be identified with such a state, and in this case the theoretical ratio Eq. (11) (identifying $D^{*}(2600)^{0}$ with $\left.\tilde{D}^{* 0}\right)$ would be

$$
\frac{B R\left(D^{* 0}(2600) \rightarrow D^{+} \pi^{-}\right)}{B R\left(D^{* 0}(2600) \rightarrow D^{*+} \pi^{-}\right)}=0.822 \pm 0.003
$$



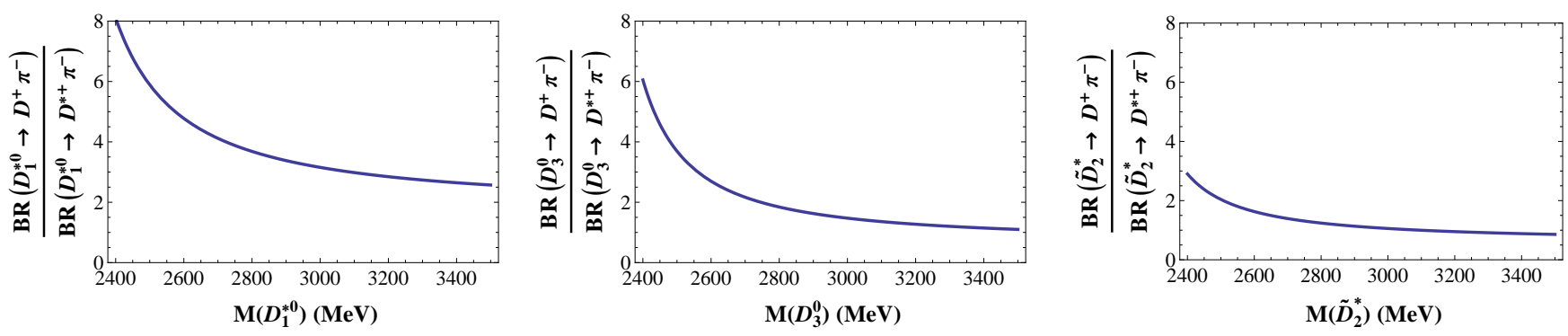

Figure 1: Ratios $\frac{B R\left(D_{1}^{*} \rightarrow D^{+} \pi^{-}\right)}{B R\left(D_{1}^{*} \rightarrow D^{*+} \pi^{-}\right)}, \frac{B R\left(D_{3} \rightarrow D^{+} \pi^{-}\right)}{B R\left(D_{3} \rightarrow D^{*+} \pi^{-}\right)}$and $\frac{B R\left(\tilde{D}_{2}^{* 0} \rightarrow D^{+} \pi^{-}\right)}{B R\left(\tilde{D}_{2}^{* 0} \rightarrow D^{*+} \pi^{-}\right)}$versus the mass of the decaying meson.

which is larger than the measurement in (11). To investigate the origin of the discrepancy, we consider the other states (up to $\ell=2$ ) for which the decays to $P M$ and $P^{*} M$ are both allowed, namely $P_{1}^{*}$ in $X, P_{3}$ in $X^{\prime}$ and $\tilde{P}_{2}^{*}$ in $\tilde{T}$. In Fig 1 we plot the ratios $\frac{B R\left(D_{1}^{* 0} \rightarrow D^{+} \pi^{-}\right)}{B R\left(D_{1}^{* 0} \rightarrow D^{*+} \pi^{-}\right)}, \frac{B R\left(D_{3}^{0} \rightarrow D^{+} \pi^{-}\right)}{B R\left(D_{3}^{0} \rightarrow D^{*+} \pi^{-}\right)}$and $\frac{B R\left(\tilde{D}_{2}^{* 0} \rightarrow D^{+} \pi^{-}\right)}{B R\left(\tilde{D}_{2}^{* 0} \rightarrow D^{*+} \pi^{-}\right)}$versus the mass of the decaying meson. The ratio exceeds 1 both for $D_{3}$ and for $D_{1}^{*}$, while in the case of $\tilde{D}_{2}^{* 0}$ it could be smaller than 1 only for a large mass, $M\left(\tilde{D}_{2}^{* 0}\right)>3100 \mathrm{MeV}$. Instead, a quark model prediction for the mass for this meson is $M\left(\tilde{D}_{2}^{* 0}\right)=3035 \mathrm{MeV}$ [20]. Therefore, no quantum number assignment to a meson with a mass of about $2600 \mathrm{MeV}$ is able to reproduce the measurement (11). A possible conclusion is that there is a violation of the HQ symmetry in the decays of $D^{* 0}(2600)$.

Let us proceed with the other resonances. Identifying $D^{*}(2760)$ with $D_{3}$ and $D(2750)$ with its spin partner $D_{2}^{\prime *}$, we obtain for the ratio (12)

$$
\left.\frac{B R\left(D^{* 0}(2760) \rightarrow D^{+} \pi^{-}\right)}{B R\left(D^{* 0}(2750) \rightarrow D^{*+} \pi^{-}\right)}\right|_{X^{\prime} \text { doublet }}=0.660 \pm 0.001
$$

which is close to the experimental result. In the hypothesis that $\left(D(2750), D^{*}(2760)\right)$ fill the $\left(\tilde{D}_{1}^{\prime}, \tilde{D}_{2}^{*}\right)$ doublet, we obtain $\left.\frac{B R\left(D^{* 0}(2760) \rightarrow D^{+} \pi^{-}\right)}{B R\left(D^{* 0}(2750) \rightarrow D^{*+} \pi^{-}\right)}\right|_{\tilde{T} \text { doublet }}=0.563 \pm 0.001$. Considering the uncertainty in (11), both these results agree with the measurement ${ }^{2}$.

The last resonance to discuss in Table VII is $D_{s J}(2860)$, for which we support the identification with $D_{s 3}$. However, the ratios in Table VII do not compare favorably with the measurement in Eq.(10) [19], and this requires an explanation. In Section \س we have predicted that the spin partner of $D_{s J}(2860)$ has mass $M\left(D_{s 2}^{* \prime}\right)=2851 \pm 7 \mathrm{MeV}$. Hence, these two states are very close to each other, and the experimental resolution in the common $D^{*} K$ decay channel could be difficult. A possible consequence is that the measurement in Eq.(10), which at first sight is attributed only to $D_{s J}(2860)$, might be contaminated by the decay $D_{s 2}^{* \prime} \rightarrow D^{*} K$, and what is actually measured is the number of final $D^{(*)} K$ pairs produced from both the states:

$$
\bar{R}(2860)=\frac{\Gamma\left(D_{s J}(2860) \rightarrow D^{*} K\right)+\Gamma\left(D_{s 2}^{* \prime}(2851) \rightarrow D^{*} K\right)}{\Gamma\left(D_{s J}(2860) \rightarrow D K\right)}
$$

The prediction for this ratio, considering the two contributions, is

$$
\bar{R}(2860)=0.99 \pm 0.05
$$

which agrees with the datum Eq. $(10)^{3}$.

The various ratios of decay rates for the beauty mesons can be predicted, using the observed masses of the two $2^{+}$ states in the $s_{\ell}^{P}=3 / 2^{+}$doublet and the predicted masses in Table $\left.\bar{V}\right]$ for the other states. The results are collected in

\footnotetext{
2 The ratios (33134) have also been computed in Ref. 34]. The result for the ratio in Eq. (33) agrees with ours, while for Eq. 34] the value 0.80 has been obtained. The case of $D^{* 0}(2760)$ belonging to the doublet $\tilde{T}$ has not been considered in that study.

${ }^{3}$ In [35] it was proposed that two overlapping structures with $J^{P}=0^{+}$and $J^{P}=2^{+}$exist in the mass range about $2860 \mathrm{MeV}$, identified with the $\mathrm{n}=2$ scalar and tensor $c \bar{s}$ states, respectively.
} 
TableVII The last line in the Table corresponds to the assignment of $D_{s J}(2860)$ and $D^{*}(2760)$ to the $X^{\prime}, s_{\ell}^{P}=5 / 2^{-}$ doublet; if these two states belong to the $\tilde{T}$ doublet, for the corresponding beauty mesons we predict $R_{\pi}=1.15 \pm 0.03$ for $\tilde{B}_{2}^{*}$, and $R_{K}=1.06 \pm 0.01, R_{\eta}=0.160 \pm 0.003, R_{\eta}^{*}=0.135 \pm 0.004$ for $\tilde{B}_{s 2}^{*}$. Therefore, the ratios $R_{\pi}$ and $R_{K}$ can be used to distinguish between the two assignments.

\section{STRONG COUPLING CONSTANTS AND DECAY WIDTHS}

The measurements of the meson widths allow us to determine the effective coupling constants. Since for mesons in the same doublet one should obtain the same result in the HQ limit, a comparison among the results tests the heavy quark symmetry and the quantum number assignment to the decaying state. From the obtained coupling constants, predictions for not yet observed states follow.

- $g$

The strong transition among states in the $H$ doublet is governed by the coupling $g$. Actually, only charmed mesons undergo real $D_{(s)}^{*} \rightarrow D \pi(K)$ transitions, the corresponding beauty modes being kinematically forbidden. The coupling constant plays an important role in processes in which the $B^{*} B \pi$ vertex is involved; at present, there is a single experimental determination of the $D^{* \pm}$ width [36] : $\Gamma\left(D^{* \pm}\right)=96 \pm 4 \pm 22 \mathrm{KeV}$, corresponding to $g=0.64 \pm 0.075$, a value which is larger than the theoretical results obtained in the HQ limit [37 39].

- $h$

The coupling constant $h$ controls the decays $S \rightarrow H M$. We can use data on the members of the $c \bar{q}$ doublet $S$, with $q=u, d$. However, the case $q=s$ corresponds to $\left(D_{s 0}^{*}(2317), D_{s 1}^{\prime}(2460)\right)$ and, as we have already discussed, the strong decays of these particles cannot be accounted by the effective Lagrangian (13) which describes isospin conserving modes. Therefore, we only consider the doublet $\left(D_{0}^{*}(2400), D_{1}^{\prime}(2430)\right)$. The values of $h$ obtained from their widths in Table II and using the rates in Eqs.(18) and (19), are: $h=0.61 \pm 0.07$ from $D_{0}^{* 0}(2400)$, $h=0.50 \pm 0.06$ from $D_{0}^{* \pm}(2400)$ and $h=0.8 \pm 0.2$ from $D_{1}^{\prime 0}(2430)$. The weighted average is

$$
h=0.56 \pm 0.04 \text {. }
$$

This result nicely agrees with the QCD sum rule outcome Ref. 38] and with the lattice QCD determination in Ref. [39]. For beauty mesons, using the predicted masses together with (37), we obtain the widths quoted in Table VI]

- $h^{\prime}$

The $T \rightarrow H M$ decays (with mesons in the doublet $T$ having $n=1$ ) are described by the coupling constant $h^{\prime}$. Using the widths in Table II together with Eqs.(20), (21) and (22), we obtain $h^{\prime}=0.56 \pm 0.03$ (from $D_{1}^{0}(2420)$ ), $h^{\prime}=0.54 \pm 0.065$ (from $\left.D_{1}^{ \pm}(2420)\right), h^{\prime}=0.43 \pm 0.01\left(\right.$ from $\left.D_{2}^{* 0}(2460)\right), h^{\prime}=0.37 \pm 0.03\left(\right.$ from $D_{2}^{* \pm}(2460)$ ) and $h^{\prime}=0.48 \pm 0.035$ (from $\left.D_{s 2}^{*}(2573)\right)$. The weighted average is

$$
h^{\prime}=0.43 \pm 0.01 .
$$

This translates into a prediction for the full width of $D_{s 1}(2536): \Gamma\left(D_{s 1}(2536)\right)=0.305 \pm 0.002 \mathrm{MeV}$. A recent determination provided by BaBar collaboration [40], quoted in Table III, is larger than our result, possibly signalling a mixing with the other axial-vector state $D_{s 1}^{\prime}(2460)$ [41].

In the case of beauty, in the $T$ doublet only the width of the $B_{2}^{* 0}$ meson has been measured, with the result $h^{\prime}=0.36 \pm 0.09$. Using this value, the predictions in Table VI follow for the other beauty resonances in $T$. The two beauty-strange states are very narrow, and they decay to $B^{*} K$ (the $B_{s 1}(5830)$ ) and to $B K, B^{*} K$ (the $\left.B_{s 2}^{*}(5840)\right)$ with a tiny phase space $\left(M_{B K} \simeq 5777 \mathrm{MeV}\right.$ and $\left.M_{B^{*} K} \simeq 5823 \mathrm{MeV}\right)$.

It is interesting to compare the results obtained from charm and beauty sectors for such a coupling constant, which in the HQ limit should coincide. Including $1 / m_{Q}$ effects, one may write: $h^{\prime}\left(m_{Q}\right)=h_{\text {asymp }}^{\prime}\left(1+a / m_{Q}\right)$. Identifying the value in (38) with $h^{\prime}\left(m_{c}\right)$ (and $m_{c} \simeq 1.35 \mathrm{GeV}$ ) and the above $h^{\prime}\left(m_{b}\right)$ (with $m_{b} \simeq 4.8 \mathrm{GeV}$ ), we find $h_{\text {asymp }}^{\prime}=0.33$ and $a=0.13 \mathrm{GeV}$. The value from the beauty data is close to the asymptotic one, while in the case of charm the correction for the coupling is of $\mathcal{O}(30 \%)$.

- $\tilde{g}$

The constant $\tilde{g}$ governs the decays $\tilde{H} \rightarrow H M$, with $\tilde{H}$ the doublet comprising the radial excitations of $H$. Observed states that fit in such a doublet, with and without strangeness, are the two resonances $D(2550), D^{*}(2600)$ 
and the strange one $D_{s 1}^{*}(2700)$. From their measured widths we obtain $\tilde{g}=0.35 \pm 0.03($ from $D(2550)$ ), $\tilde{g}=0.23 \pm 0.02\left(\right.$ from $\left.D^{*}(2600)\right), \tilde{g}=0.31 \pm 0.04$ (from $D_{s 1}^{*}(2700)$ ), with weighted average

$$
\tilde{g}=0.28 \pm 0.015 \text {. }
$$

We can predict the full width of the spin partner of $D_{s 1}^{*}(2700)$ using the mass fixed in Sec. III, as reported in Table $\nabla$. The predictions for the widths of the beauty resonances belonging to $H^{\prime}$ are collected in Table VI

According to the interpretation of the remaining states [of $\left(D(2750), D^{*}(2760)\right)$, of $D_{s J}(2860)$ and its spin partner that we denote with $D_{s J}(2851)$ ], we could determine one more constant. However, for such states other strong decay modes besides those considered here are possible. Kinematically allowed transitions with the emission of a light vector meson are

$$
\begin{gathered}
D(2750), D^{*}(2760) \rightarrow D \rho, D \omega \\
D_{s J}(2851), D_{s J}(2860) \rightarrow D K^{*} .
\end{gathered}
$$

These decays are possible both for states filling the $X^{\prime}, J^{P}=\left(2^{-}, 3^{-}\right)$doublet, both for mesons belonging to the doublet $\tilde{T}$ with $J^{P}=\left(1^{+}, 2^{+}\right)$. In the first case these processes occur in $f$-wave, in the second one in $d$ - wave. The $2^{-}$ state could decay also in $p$ - wave, and the $1^{+}$meson in $s$-wave, but at a next-to-leading order in the HQ expansion. The strange meson decays in (40) are severely phase-space suppressed.

Other kinematically allowed transitions are the decays to a member of one of the excited doublets $\tilde{H}, S, T$ and a light pseudoscalar meson. In the case of the $X^{\prime}$ doublet, allowed $f$-wave decay modes are

$$
\begin{aligned}
D(2750) & \rightarrow D_{0}^{*}(2400) \pi, D_{2}^{*}(2460) \pi \\
D^{*}(2760) & \rightarrow D_{1}^{\prime}(2430) \pi, D_{1}(2429) \pi, D_{2}^{*}(2460) \pi .
\end{aligned}
$$

Decays to the members of the doublet $\tilde{H}$, identified with $\left(D(2550), D^{*}(2600)\right)$ are possible, however, the available phase space is almost closed for $D^{*}(2600)$, and is about $60-70 \mathrm{MeV}$ for $D(2550)$.

In the case of the $\tilde{T}$ doublet, allowed decay modes are

$$
\begin{aligned}
D(2750) & \rightarrow D_{0}^{*}(2400) \pi, D_{1}^{\prime}(2430) \pi, D_{1}(2420) \pi, D_{2}^{*}(2460) \pi, \\
D^{*}(2760) & \rightarrow D_{1}^{\prime}(2430) \pi, D_{1}(2420) \pi, D_{2}^{*}(2460) \pi,
\end{aligned}
$$

which proceed in $p$-wave. It is important to notice that the transitions $D(2750) \rightarrow D_{1}^{\prime}(2430) \pi, D_{1}(2420) \pi$ are allowed only if $D(2750)$ has $J^{P}=1^{+}, n=2$, the alternative possibility considered in this paper. Therefore, experimental study of this decay mode is useful to establish the correct classification.

All the listed modes have small phase space. Therefore, we can adopt the same strategy used to determine $h, h^{\prime}$, $\tilde{g}$ to fix the constant that determines the strong decays of $\left(D(2750), D^{*}(2760)\right)$ and $D_{s J}(2860)$, that depends on the doublet in which we place them. The obtained values should be viewed as upper bounds on the couplings, since we neglect suppressed decay modes.

In the classification of $D_{s J}(2860)$ as the $J^{P}=3^{-}$state belonging to the $X^{\prime}$ doublet, the resonances $\left(D(2750), D^{*}(2760)\right)$ fill the corresponding non strange doublet. From their mass and width we obtain the coupling $k=k_{1}+k_{2}: k=0.58 \pm 0.05($ from $D(2750)), k=0.39 \pm 0.02\left(\right.$ from $\left.D^{*}(2760)\right), k=0.41 \pm 0.03\left(\right.$ from $D_{s J}(2860)$ ). The average is

$$
k=0.42 \pm 0.02 \text {. }
$$

Using this result we predict the full widths of the $D_{s 2}^{\prime *}$, the spin partner of $D_{s J}(2860)$, and of the analogous beauty states [keeping in mind that also for beauty other decay modes are possible, the analogous of those in Eqs. (4041)]. Using the masses in Sec. III we obtain the results quoted in Tables V and VI]

Alternatively, if $D_{s J}(2860)$ is the $n=2, J^{P}=2^{+}$state, i.e. the first radial excitation of $D_{s 2}^{*}(2573)$, and $(D(2750), D(2760))$ the non strange members of the $\tilde{T}$ doublet of radial excitations of $T$, we can fix the constant $\tilde{h}^{\prime}$ governing the decays $\tilde{T} \rightarrow H M$. The results are $\tilde{h}^{\prime}=0.23 \pm 0.02($ from $D(2750)), \tilde{h}^{\prime}=0.18 \pm 0.01($ from $D(2760)$ ), $\tilde{h}^{\prime}=0.17 \pm 0.01\left(\right.$ from $D_{s J}(2860)$ ), with average

$$
\tilde{h}^{\prime}=0.18 \pm 0.01
$$

In this case, the spin partner of $D_{s J}(2860)$ is $\tilde{D}_{s 1}$ with $J^{P}=1^{+}$, and its full width is $\Gamma\left(\tilde{D}_{s 1}\right)=30 \pm 3 \mathrm{MeV}$, while for the $\tilde{T}$ beauty states we predict: $\Gamma\left(\tilde{B}_{1}\right)=96 \pm 7 \mathrm{MeV}, \Gamma\left(\tilde{B}_{2}^{*}\right)=111 \pm 8.5 \mathrm{MeV}, \Gamma\left(\tilde{B}_{s 1}\right)=67 \pm 5 \mathrm{MeV}$ and $\Gamma\left(\tilde{B}_{s 2}^{*}\right)=83 \pm 6.4 \mathrm{MeV}$ [neglecting the modes analogous to (40) and (42)].

Finally, we quote our results for the widths of the $s_{\ell}^{P}=3 / 2^{+}$beauty states: $\Gamma\left(B_{1}\right)=13.6 \pm 0.6 \mathrm{MeV}, \Gamma\left(B_{s 1}\right)=$ $0.016 \pm 0.002 \mathrm{MeV}$ and $\Gamma\left(B_{s 2}^{*}\right)=0.9 \pm 0.1 \mathrm{MeV}$. 


\section{CONCLUSIONS}

The heavy quark symmetry is a powerful tool for the analysis of the properties of hadrons with a single heavy quark. Using ideas and methods based on this symmetry, we have proposed a classification of all the observed $c \bar{q}$ and $b \bar{q}$ mesons in doublets, as shown in Table \ determining a set of mass parameters from data. Moreover, we have fixed several effective coupling constants governing the strong transitions into the lightest heavy quark doublet. With these inputs, we have predicted the mass and width of two not yet observed $c \bar{s}$ resonances, reported in Table $\mathrm{V}$. The contribution of the new state $D_{s J}(2851)$ has been advocated to explain a discrepancy between the observed ratio of $D^{*} K / D K$ yield in the invariant mass range around $2850-2870 \mathrm{MeV}$ and the theoretical result obtained assuming only the contribution of $D_{s J}(2860)$ to this observable. The other main experimental observables in the charm sector are reproduced, with the exception of the first ratio in (11) for the $D^{*}(2600)$. Finally, we have predicted the properties of the not yet observed $b \bar{q}$ mesons: the confirmation of such predictions is expected in the very near future from the experiments at the LHC.

\section{Acknowledgement}

We thank D. Milanes and A. Palano for discussions. This work is supported in part by the Italian MIUR Prin 2009.

[1] For reviews see: M. Neubert, Phys. Rept. 245, 259 (1994); F. De Fazio, in At the Frontier of Particle Physics/Handbook of $Q C D$, ed. by M. Shifman (World Scientific, Singapore, 2001), page 1671, arXiv:hep-ph/0010007.

[2] N. Isgur and M. B. Wise, Phys. Lett. B 237, 527 (1990).

[3] N. Isgur and M. B. Wise, Phys. Rev. Lett. 66, 1130 (1991); M. Lu, M. B. Wise and N. Isgur, Phys. Rev. D 45, 1553 (1992).

[4] M. B. Wise, Phys. Rev. D 45, 2188 (1992); G. Burdman and J. F. Donoghue, Phys. Lett. B 280, 287 (1992); P. L. Cho, Phys. Lett. B 285, 145 (1992); Phys. Rev. D 46, 1148 (1992) [Erratum-ibid. D 55, 5851 (1997)]; R. Casalbuoni, A. Deandrea, N. Di Bartolomeo, R. Gatto, F. Feruglio and G. Nardulli, Phys. Lett. B 299, 139 (1993).

[5] For a review see: A. V. Manohar and M. B. Wise, Camb. Monogr. Part. Phys. Nucl. Phys. Cosmol. 10, 1 (2000).

[6] A. F. Falk and T. Mehen, Phys. Rev. D 53 (1996) 231.

[7] J. Beringer et al. (Particle Data Group), Phys. Rev. D 86, 010001 (2012) and http://pdg.lbl.gov/

[8] S. Anderson et al. [CLEO Collaboration], Nucl. Phys. A663, 647 (2000).

[9] K. Abe et al. [Belle Collaboration], Phys. Rev. D 69, 112002 (2004).

[10] J. M. Link et al. [FOCUS Collaboration], Phys. Lett. B586, 11 (2004).

[11] B. Aubert et al. [BABAR Collaboration], Phys. Rev. Lett. 90, 242001 (2003); Y. Mikami et al. [Belle Collaboration], Phys. Rev. Lett. 92, 012002 (2004); K. Abe et al., Phys. Rev. Lett. 92, 012002 (2004); D. Besson et al. [CLEO Collaboration], Phys. Rev. D 68, 032002 (2003); L. Benussi [FOCUS Collaboration], Int. J. Mod. Phys. A 20, 549 (2005); B. Aubert et al. [BABAR Collaboration], Phys. Rev. D69, 031101 (2004); P. Krokovny et al. [Belle Collaboration], Phys. Rev. Lett. 91, 262002 (2003); P. Krokovny, AIP Conf. Proc. 717, 475 (2004).

[12] P. Colangelo, F. De Fazio and A. Ozpineci, Phys. Rev. D 72, 074004 (2005).

[13] T. E. Browder, S. Pakvasa and A. A. Petrov, Phys. Lett. B 578, 365 (2004).

[14] B. Aubert et al. [BABAR Collaboration], Phys. Rev. Lett. 97, 222001 (2006).

[15] J. Brodzicka et al. [Belle Collaboration], Phys. Rev. Lett. 100, 092001 (2008).

[16] R. Aaij et al. [ LHCb Collaboration], arXiv:1207.6016 [hep-ex].

[17] B. Aubert et al. [BABAR Collaboration], Phys. Rev. D 80, 092003 (2009).

[18] P. Colangelo, F. De Fazio, S. Nicotri and M. Rizzi, Phys. Rev. D 77, 014012 (2008).

[19] P. Colangelo, F. De Fazio and S. Nicotri, Phys. Lett. B 642, 48 (2006).

[20] M. Di Pierro and E. Eichten, Phys. Rev. D 64, 114004 (2001).

[21] P. Colangelo and F. De Fazio, Phys. Rev. D 81, 094001 (2010).

[22] P. del Amo Sanchez et al. [The BABAR Collaboration], Phys. Rev. D 82, 111101 (2010).

[23] S. Godfrey and N. Isgur, Phys. Rev. D 32, 189 (1985).

[24] R. Akers et al. [OPAL Collaboration], Z. Phys. C 66, 19 (1995).

[25] P. Abreu et al. [DELPHI Collaboration], Phys. Lett. B 345, 598 (1995); D. Buskulic et al. [ALEPH Collaboration], Z. Phys. C 69, 393 (1996); R. Barate et al. [ALEPH Collaboration], Phys. Lett. B 425, 215 (1998); M. Acciarri et al. [L3 Collaboration], Phys. Lett. B 465, 323 (1999).

[26] V. M. Abazov et al. [D0 Collaboration], Phys. Rev. Lett. 99, 172001 (2007).

[27] T. Aaltonen et al. [CDF Collaboration], Phys. Rev. Lett. 102, 102003 (2009).

[28] T. Aaltonen et al. [CDF Collaboration], Phys. Rev. Lett. 100, 082001 (2008).

[29] V. M. Abazov et al. [D0 Collaboration], Phys. Rev. Lett. 100, 082002 (2008).

[30] LHCB Collaboration, LHCb-CONF-2011-053 (2011). 
[31] P. Colangelo and F. De Fazio, Phys. Lett. B 570, 180 (2003).

[32] P. Colangelo, F. De Fazio and R. Ferrandes, Mod. Phys. Lett. A 19, 2083 (2004).

[33] P. Colangelo, F. De Fazio and R. Ferrandes, Phys. Lett. B 634, 235 (2006).

[34] Z. G. Wang, Phys. Rev. D 83, 014009 (2011).

[35] E. van Beveren and G. Rupp, Phys. Rev. D 81, 118101 (2010).

[36] A. Anastassov et al. [CLEO Collaboration], Phys. Rev. D 65, 032003 (2002).

[37] P. Colangelo, G. Nardulli, A. Deandrea, N. Di Bartolomeo, R. Gatto and F. Feruglio, Phys. Lett. B 339, 151 (1994); P. Colangelo, F. De Fazio and G. Nardulli, Phys. Lett. B334, 175 (1994); V. M. Belyaev, V. M. Braun, A. Khodjamirian and R. Ruckl, Phys. Rev. D 51, 6177 (1995); D. Becirevic, B. Blossier, E. Chang and B. Haas, Phys. Lett. B 679, 231 (2009).

[38] P. Colangelo, F. De Fazio, G. Nardulli, N. Di Bartolomeo and R. Gatto, Phys. Rev. D 52 (1995) 6422; P. Colangelo and F. De Fazio, Eur. Phys. J. C 4 (1998) 503.

[39] D. Becirevic, E. Chang and A. L. Yaouanc, arXiv:1203.0167 [hep-lat].

[40] J. P. Lees et al. [The BABAR Collaboration], Phys. Rev. D 83, 072003 (2011).

[41] V. Balagura et al. [Belle Collaboration], Phys. Rev. D 77, 032001 (2008). 